\title{
CAPÍTULO 15
}

\section{MECANISMO DE ACCIÓN DEL SABOR Y DEL GUSTO UMAMI EN EL CEREBRO}

Edmund T. Rolls

\section{RESUMEN}

El mecanismo de acción del umami en la corteza cerebral revela la razón que lo hace agradable y apetitoso. El carácter agradable del umami refleja y está correlacionado con el procesamiento en el área gustativa cortical secundaria, (corteza orbitofrontal) y con el área gustativa cortical terciaria (corteza cingulada anterior), mientras el procesamiento en el área cortical primaria del gusto (insular), refleja propiedades físicas, como la intensidad. Sin embargo, el glutamato puro, por sí solo, no tiene un sabor muy agradable como estímulo gustativo y no actúa sinérgicamente con otros gustos (dulce, salado, amargo y ácido). Cuando el glutamato se administra en combinación con un estímulo olfatorio armónico, por ejemplo con un rico aroma (vegetal), el sabor resultante, formado por una convergencia de las vías gustativas y olfativas en la corteza orbitofrontal, puede ser mucho más agradable. Esta palatabilidad se refleja en una activación de la corteza orbitofrontal media y de la corteza cingulada pregenual mucho mayor que la suma de las activaciones de los componentes gustativos y olfatorios presentados separadamente. Además, existe una relación recíproca entre las activaciones en estas regiones del cerebro con la apetencia y plenitud del gusto, 
como también existe esa relación entre la armonía del gusto y los componentes olfatorios. A nivel conceptual, se propone que el umami puede ser pensado como un sabor rico y delicioso, producido por la combinación del gusto del glutamato con un estímulo olfatorio armónico, es decir con un aroma delicioso. El glutamato es, entonces, un realzador del sabor por la forma como puede combinarse supralinealmente con olores armónicos, en áreas corticales donde las vías del gusto y del olfato convergen más allá de los receptores. La modulación de las regiones corticales en la corteza orbitofrontal, entre ellas el área de la atención y el área cognitiva, también contribuyen a la palatabilidad del umami.

\section{INTRODUCCIÓN}

El gusto al que se refiere la palabra japonesa umami ha sido reconocido como el "quinto gusto" (Kawamura \& Kare, 1987; Rolls, 2000). Además del dulce, salado, amargo y ácido, el umami se traduce en lo que a veces se describe como un gusto de proteína. De hecho, métodos de análisis de escala multidimensional en humanos (Yamaguchi \& Kimizuka, 1979) han mostrado que el gusto del glutamato (tal como su sal de sodio, glutamato monosódico/GMS) no puede ser comparado con ninguno de los cuatro gustos básicos. Aún más, se han encontrado receptores específicos del gusto para el glutamato (Chaudhari et al., 2000; Zhao et al., 2003; Maruyama et al., 2006). El gusto umami se encuentra en una diversidad de alimentos ricos en glutamato, entre ellos el pescado, la carne, la leche, los tomates, en algunas verduras y es potenciado por algunos 5'-ribonucleótidos (inosina y guanosina incluidos) (Yamaguchi, 1967; Rifkin \& Bartoshuk, 1980). Estos ribonucleótidos están presentes, por ejemplo, en carnes y en algunos pescados (Yamaguchi \& Ninomiya, 2000). La mezcla de esos componentes es responsable por el sabor delicioso característico de muchos alimentos.

En este capítulo, se resumen los hallazgos sobre cómo el gusto umami está representado por las neuronas perfectamente sintonizadas que se encuentran en las áreas corticales del gusto en los monos; y luego se hacen conjeturas basado en estos estudios para examinar la representación del gusto umami en el cerebro humano. La pregunta que subyace, entonces, es ¿qué mecanismo hace posible que el umami sea percibido sensorialmente como un gusto agradable y apetecible?. La importancia de entender esta interrogante se justifica, en parte, debido a que el umami es un indicador sensorial clave de alimentos que contienen proteínas $\mathrm{y}$, por ende, una señal importante que apunta hacia alimentos que ayudan a mantener una dieta nutricionalmente apropiada. 


\section{ESTUDIOS NEURONALES EN MONOS}

Con el objetivo de entender la forma cómo el apetito y la ingesta de alimentos son controlados por el cerebro humano, así como también los desórdenes relacionados con el apetito y la alimentación, se han desarrollado diversos estudios, tanto en primates como en humanos, acerca de los mecanismos neuronales involucrados en la regulación de estos eventos (Rolls, 2005; Rolls, 2007a; Rolls, 2007b; Rolls, 2008a; Rolls \& Grabenhorst, 2008). Una de las razones del empleo de primates como animales de experimentación en algunos de estos estudios, se debe al hecho de que su sistema gustativo está organizado anatómica y fisiológicamente de manera diferente al de los no-primates (Norgren, 1984; Rolls \& Scott, 2003; Rolls, 2009). A diferencia de los roedores, por ejemplo, en los monos no existe un conjunto de vías subcorticales a partir del tronco cerebral. En su lugar, hay una conexión obligatoria del núcleo del tracto solitario del tálamo gustativo, con la corteza gustativa (Norgren, 1984; Rolls \& Scott, 2003). Esto torna el sistema gustativo de los primates más fácil de analizar, mucho más que el de humanos en relación al procesamiento del gusto que ocurre en la corteza gustativa primaria y prosigue luego hacia otras áreas (Figura 15.1). En cambio, en los roedores existen salidas desde la zona (gustativa) del tronco cerebral a los sistemas subcorticales. Además, el comer hasta la saciedad reduce el grado de respuesta de las neuronas gustativas en la corteza gustativa (orbitofrontal) secundaria, pero no en la corteza gustativa (insular) primaria. Por otro lado, los efectos de la saciedad en el procesamiento del gusto son encontrados incluso en el núcleo del tracto solitario de roedores (Rolls \& Scott, 2003), lo que torna al sistema gustativo de roedores más complejo de analizar. 


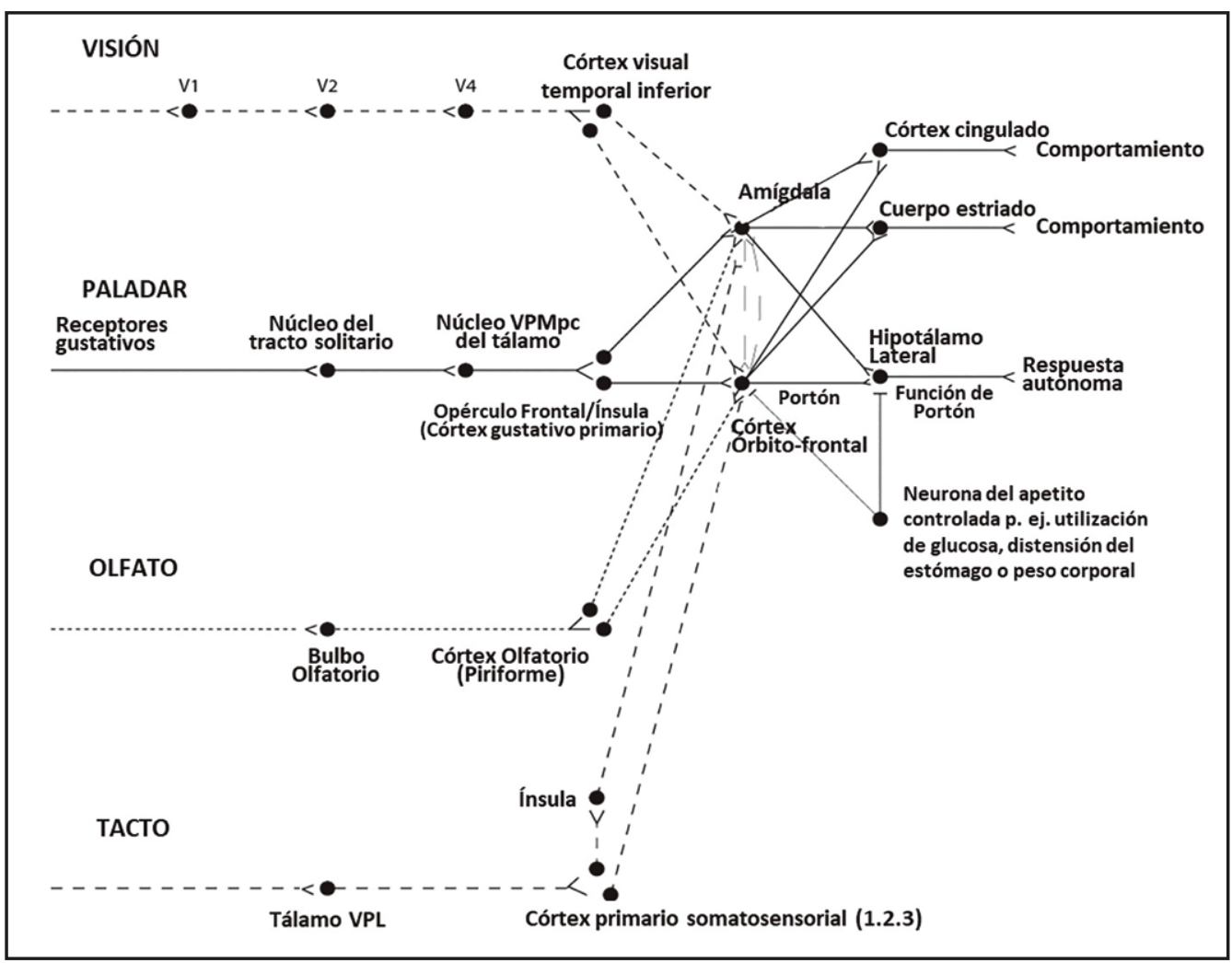

Figura 15.1 - Esquema de las rutas olfativas y gustativas en los primates, incluyendo humanos; se muestra la convergencia en un mismo espacio y la confluencia con las rutas visuales. El hambre modula la respuesta de las representaciones en la corteza orbitofrontal del gusto, aroma, textura y visión del alimento (indicado por la función porta), y la corteza orbitofrontal donde son representados la palatabilidad y el placer proporcionado por el alimento. VPMpc -núcleo talámico ventroposteromedial; V1, V2, V4 - áreas corticales visuales (tasolfpaths2.eps).

Se ha demostrado que en la corteza orbitofrontal de primates, hay una región de la corteza gustativa secundaria que se conecta con la corteza gustativa primaria en la ínsula y en el opérculo frontal adyacente (Scott et al., 1986; Yaxley et al., 1990; Baylis et al., 1995; Scott \& Plata-Salaman, 1999). En esta región, las neuronas son activadas por el sabor del alimento (Rolls et al., 1990; Rolls, 2008a) (Figura 15.1). Estas neuronas gustativas de la corteza orbitofrontal pueden ser finamente sintonizadas con el estímulo gustativo (Rolls et al., 1990). Aún más, esa actividad neuronal está relacionada a la recompensa del alimento, ya que las neuronas responden al gusto del alimento solamente cuando el mono está con hambre (Rolls et al., 1989). Estas neuronas, muestran sus efectos en el proceso de saciedad sensorial específica, un mecanismo 
primordial en el control de la alimentación, el cual tiene implicaciones importantes para el control del apetito y la ingesta de alimentos (Rolls et al., 1989; Critchley \& Rolls, 1996b; Rolls, 2005; Rolls, 2007b). La corteza orbitofrontal está involucrada en el control de la alimentación porque esta es la primera parte del sistema gustativo de los primates donde ocurren las respuestas neuronales al gusto del alimento durante el periodo de la sensación de hambre, pero no después de la saciedad (Rolls et al., 1988; Yaxley et al., 1988; Rolls et al., 1989; Critchley \& Rolls, 1996b; Rolls, 2007b).

\section{NEURONAS ESPECÍFICAMENTE REGULADAS POR EL GLUTAMATO}

Para investigar si el gusto umami opera en el sistema gustativo de primates a través de redes de información diferentes a las vías de procesamiento de los otros gustos (dulce, salado, amargo y ácido), Baylis \& Rolls (1991) analizaron las respuestas de 190 neuronas gustativas sensibles, situadas en la corteza gustativa primaria y en el área adyacente de la corteza orbitofrontal de monos en estado de vigilia. Este estudio encontró la presencia de neuronas simples, ajustadas para responder mejor al glutamato (GMS) (gusto umami) así como también fueron halladas otras células que respondían mejor a la glucosa (dulce), al cloruro de sodio $(\mathrm{NaCl})$ (salado), al ácido clorhídrico $(\mathrm{HCl})$ (ácido), e hidocloruro de quinina (Q-HCl) (amargo). Dentro de la población de neuronas, la capacidad de respuesta al glutamato fue pobre, en relación con la respuesta al $\mathrm{NaCl}$. De este modo, la representación de la actividad neuronal del glutamato mostró ser diferente del perfil del $\mathrm{NaCl}$. Aún más, el perfil del glutamato mostró también ser diferente del perfil de cada uno de los otros cuatro gustos, en una medida aproximada a la diferencia observada entre los cuatro gustos básicos, comparados entre sí. Estos resultados fueron demostrados por medición multidimensional y análisis de grupos. Baylis \& Rolls (1991) concluyeron que, en las áreas corticales gustativas de primates, el glutamato, que produce el gusto umami en humanos, está también representado como lo están los otros gustos producidos por la glucosa (dulce), el $\mathrm{NaCl}$ (sal), el $\mathrm{HCl}$ (ácido) y la Q- $\mathrm{HCl}$ (amargo).

\section{NEURONAS QUE RESPONDEN AL GUSTO DEL GLUTAMATO MONOSÓDICO TAMBIÉN RESPONDEN AL GUSTO DEL ÁCIDO GLUTÁMICO}

Para evaluar el papel del ion glutamato en el perfil del gusto umami, Rolls et al. (1996a) realizaron una investigación neurofisiológica en la que se hicieron registros de las respuestas neuronales de la corteza orbitofrontal de monos 
utilizando estímulos con ácido glutámico. En este estudio se demostró que algunas neuronas tuvieron amplias respuestas para la sensación gustativa de ácido glutámico $0,05 \mathrm{M}$ y que las células que respondieron al ácido glutámico también respondieron al GMS. Sin embargo, estas neuronas no necesariamente tuvieron amplias respuestas a $\mathrm{HCl} 0,01 \mathrm{M}$ (el pH del ácido glutámico es de 2,1). La correlación entre las respuestas de esta población de neuronas tanto al GMS como al ácido glutámico fue 0,75. Esta semejanza fue más grande que la mayoría de las correlaciones entre los demás estímulos gustativos. Este dato refuerza la evidencia de que, en el cerebro de los primates, el gusto umami está representado separadamente de las representaciones equivalentes a los otros cuatro gustos básicos.

\section{NEURONAS QUE RESPONDEN AL GUSTO DEL GLUTAMATO MONOSÓDICO,} TAMBIÉN RESPONDEN AL GUSTO DE INOSINA MONOFOSFATO.

Dado que en la boca, la inosina 5'-monofostato (IMP) produce el gusto umami en humanos, y también puede actuar sinérgicamente con el GMS, los efectos neurofisiológicos de este compuesto fueron investigados en primates (Rolls et al., 1996b). El conjunto de estímulos gustativos [glucosa 1,0 M (G), $\mathrm{NaCl} 0,1 \mathrm{M}(\mathrm{N}), \mathrm{HCl}$ 0,01 M (H), Q-HCl 0,001 M (Q), GMS 0,1 M (GMS), e IMP 0,0001 M (IMP)] fue evaluado en secuencia aleatoria. Aunque la concentración de IMP sea considerada baja, fue escogida así debido a que en estudios preliminares en monos resultó efectiva en la producción de respuestas neuronales. En humanos, esta concentración de IMP está por debajo del umbral de detección de IMP pura (Yamaguchi, 1967). No obstante, esta es una concentración que, aparentemente, es capaz de afectar el sistema gustativo humano porque, en esta especie, está en el rango de concentración que tiene un efecto sinérgico con el GMS. Se ha demostrado que las neuronas de la corteza orbitofrontal de primates responden a concentraciones tan bajas como 0,0001 $\mathrm{M}$ de IMP, y que, típicamente, estas mismas neuronas, también responden al GMS. De hecho, en toda la población de neuronas, IMP produjo respuestas más parecidas a las inducidas por GMS que a las producidas por cualquier otro de los gustos evaluados (Rolls et al., 1996b).

\section{SACIEDAD}

Experimentos con monos, demostraron que alimentarlos hasta causar la saciedad disminuye la respuesta de las neuronas de la corteza gustativa orbitofrontal 
a los alimentos con los cuales hayan sido alimentados hasta saciar (Rolls et al., 1989; Critchley \& Rolls, 1996b). Esta modulación de las respuestas gustativas por el hambre no ha sido encontrada en la corteza gustativa primaria. Además, la disminución del grado de respuesta neuronal en la corteza gustativa secundaria es, por lo menos en parte, específica para el alimento ingerido por el mono hasta la saciedad. Esto es por lo tanto considerado como una disminución sensorial específica en las respuestas (Rolls, 2007b). Se ha investigado si la saciedad inducida por la alimentación con una solución de GMS afecta las respuestas de las neuronas gustativas de la corteza orbitofrontal al gusto del GMS y si esta respuesta es sensorialmente específica. La modulación de la capacidad de respuesta por el hambre implicaría que las neuronas estuviesen en un sistema de respuesta motivacional al alimento. La demostración de saciedad sensorial específica reforzaría la evidencia de la existencia de un mecanismo neuronal separado para la percepción del gusto umami.

Las células que respondieron al gusto del GMS, o que respondieron ante la vista del alimento (Rolls \& Baylis, 1994), fueron evaluadas antes, durante y después de alimentar a un mono con 0,1 M de GMS hasta que el comportamiento del animal evidenciara saciedad. Rolls et al. (1996a) realizaron experimentos para verificar el efecto de la saciedad en respuestas gustativas al glutamato utilizando 5 neuronas (Rolls et al., 1996b). Se encontró que algunas de estas neuronas mostraron una pequeña respuesta al gusto del glutamato, después de que este fue ingerido hasta la saciedad. No obstante, las mismas neuronas mantuvieron su capacidad de respuesta a los otros gustos. De esta forma, el valor de recompensa y el placer del gusto umami se encuentran representados en la corteza orbitofrontal (Rolls, 2001; Rolls, 2003).

Estos trabajos y otras investigaciones relacionadas han aportado las bases fundamentales para la comprensión, a nivel neuronal, del mecanismo gustativo, olfativo, táctil (textura y temperatura oral) y visual involucrado en el análisis sensorial de los alimentos. Estos estudios también han contribuido a promover el valor de recompensa explícito en las representaciones de la actividad neuronal, como muestran los efectos observables tras una alimentación hasta la saciedad; revela, por ende, el importante papel que juega la actividad neuronal en el control del apetito y de la ingesta de alimentos (Kadohisa et al., 2005; Rolls, 2005; Rolls, 2007b; Grabenhorst et al., 2008b; Rolls, 2008a; Rolls, 2009). Es necesario realizar investigaciones en humanos, mediante neuroimagen funcional, que contribuyan a la construcción de este conocimiento. 


\section{LA REPRESENTALIÓN DEL GUSTO UMAMI EN LA CORTEZA CEREBRAL HUMANA}

Utilizando imágenes de resonancia magnética funcional (fMRI) (Small et al., 1999; O’Doherty et al., 2001), de Araujo et al. (2003a) investigaron si las áreas corticales, que previamente mostraron ser activadas en humanos por los otros gustos, eran también activadas por el gusto umami, y si esta activación en áreas específicas reflejaba el sinergismo entre el GMS e IMP. En esta investigación acerca del gusto, se usó una solución control sin gusto $(25 \mathrm{mM} \mathrm{KCl}+$ 2,5 $\mathrm{mM} \mathrm{NaHCO}$ ) para comparar con la respuesta producida por el estímulo gustativo. Al utilizar este contraste, es posible medir los efectos causados por el gusto, así como también los efectos somato-sensoriales de la solución control sin gusto. De igual forma, pueden ser medidos los movimientos requeridos para la deglución de la solución al final en cada periodo de la prueba gustativa. Respuestas corticales al estímulo umami fueron investigadas en diez individuos y evaluadas a través del suministro de diferentes soluciones que contenían glucosa (1 M, como localizador), GMS (0,05 M), IMP (0,005 M) o GMS+IMP (mezclado en concentraciones iguales) (de Araujo et al., 2003a). El protocolo experimental consistió en un esquema de pruebas intercaladas en las cuales, al comienzo de un periodo aleatorio variable de 12-20 segundos, uno de los cuatro estímulos gustativos fue puesto en la boca del individuo en alícuotas de $0,75 \mathrm{~mL}$, y deglutido después de 10 segundos. Luego, al inicio del siguiente periodo, fue suministrada una solución control sin gusto. En seguida se administró otro de los estímulos gustativos, en una secuencia pseudo-aleatoria. Este ciclo de pruebas fue repetido doce veces. Los resultados mostraron claramente activaciones significativas en la corteza orbitofrontal (Wilson et al., 2002; de Araujo et al., 2003a).

Los efectos del gusto umami sobre la activación cortical, fueron demostrados en diversos grupos con diferentes estímulos prototípicos, a saber: activación cortical producida por el estímulo representativo del gusto dulce utilizando glucosa (1 M) (Figura 15.2, línea 1); activación producida por el IMP $(0,0005 \mathrm{M})$ (Figura 15.2, línea 2); y la activación con GMS (0,05 M) (Figura 15.2, línea 3). Para todos los estímulos sensoriales, se detectó activación de la corteza orbitofrontal y de la corteza gustativa insular-opercular, la cual es, presuntamente, la corteza gustativa primaria en la especie humana. La activación producida por el IMP muestra que el umami, incluso cuando la solución suministrada no contiene iones sodio, activa estas áreas de la corteza cerebral. Para analizar si existen áreas de superposición de las activaciones producidas por el estímulo umami y por la glucosa usada como un estímulo gustativo prototipo en la Figura 15.2 línea 5, se observa la conjunción de los efectos producidos por GMS, IMP, GMS+IMP, 
y glucosa. Estos resultados (de Araujo et al., 2003a) aportaron evidencias de que cuando el GMS y el IMP, que producen gusto umami, son colocados en la boca, se activan áreas corticales conocidas por su activación ante otras sustancias estimuladores del gusto (glucosa).

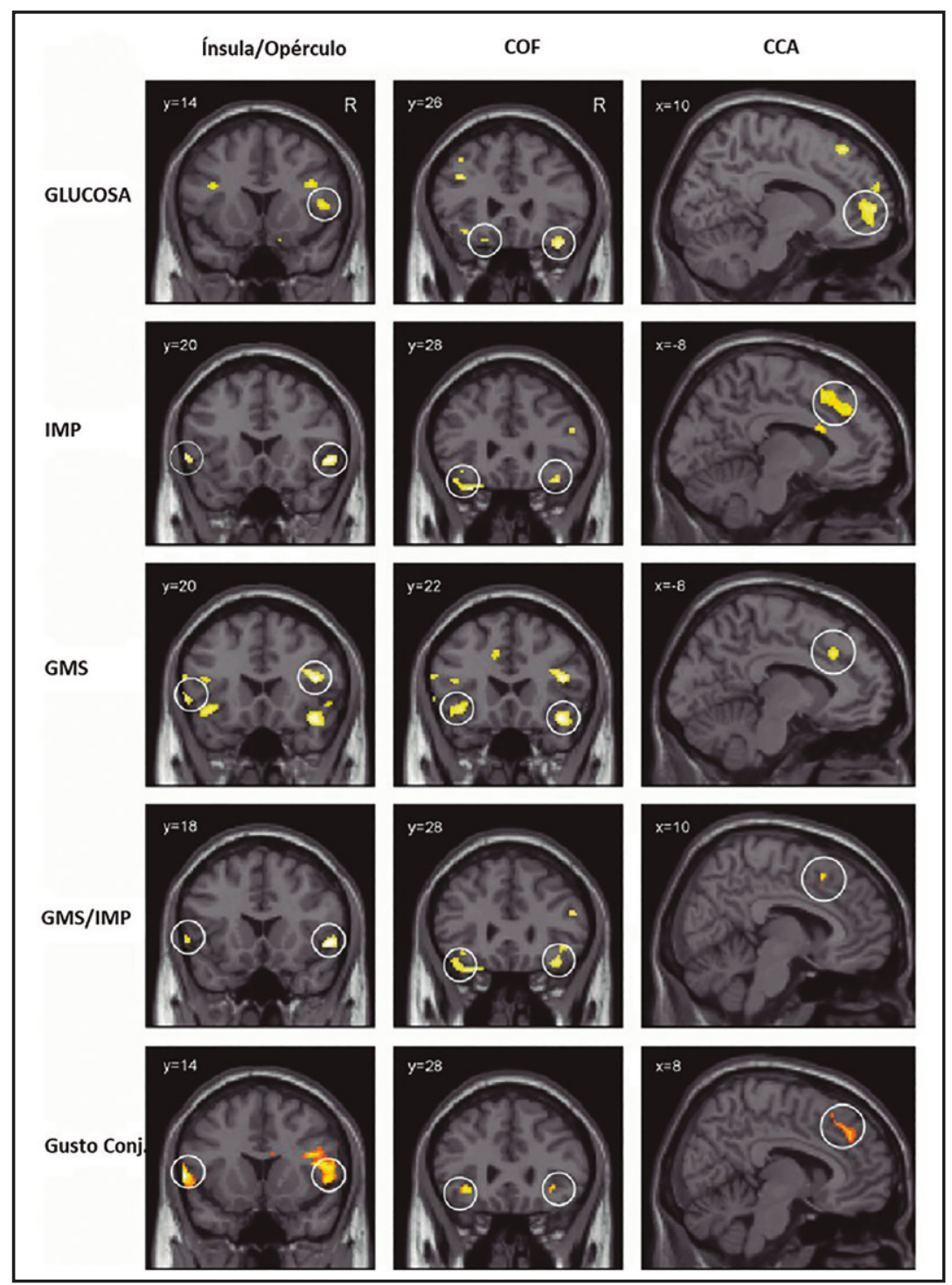

Figura 15.2 - Activaciones producidas en el ínsula/opérculo rostral, en la corteza orbitofrontal (COF) y en la corteza cingulada anterior (CCA) por glucosa (1 M), inosina-5'-monofosfato (IMP) (0,005 M), glutamato monosódico (GMS) (0,05 M), para la combinación de GMS e IMP (GMS/IMP), y la conjunción de todas las pruebas (Gusto conj.).

Fuente: de Araujo et al., 2003a. (umami2 .eps). 
Dada la evidencia de que el IMP (o su equivalente guanosina-5'-monofosfato) y el GMS pueden presentar sinergismo psicofisiológico (Rifkin \& Bartoshuk, 1980), fue de considerable interés el estudio en que de Araujo et al. (2003a) demostraron que la corteza orbitofrontal lateral anterior, región izquierda $(\mathrm{x}, \mathrm{y}, \mathrm{z}=-44,34,-18)$ presenta una propiedad aditiva supralineal ante la combinación GMS+IMP; es decir, ocurre una significativa activación, que es mayor por la combinación GMS+IMP que por la suma de los efectos del GMS e IMP, considerados separadamente (Figura 15.2). La interacción real entre el GMS e IMP puede ser expresada, en parte, en los propios receptores gustativos, o puede haber receptores de umami un tanto distintos para los diferentes probadores de umami (Chaudhari et al., 2000; Zhao et al., 2003; Maruyama et al., 2006). Sin embargo, en cualquiera de los casos, los resultados relatados por de Araujo et al. (2003a) muestran que, en la especie humana, existe una parte de la corteza orbitofrontal anterior en la cual la propiedad aditiva supralineal aparece muy fuertemente en el análisis estadístico. El hecho de que esa parte de la región de la corteza orbitofrontal humana refleja, estadísticamente, efectos supra-aditivos entre los provadores de umami, evidentes en la señal BOLD (señal dependiente del nivel de oxigenación sanguínea) indica la probabilidad de que la actividad en esta región de la corteza orbitofrontal sea especialmente relevante para la sensación perceptible del gusto umami, y para la manifestación comportamental de una preferencia por ese gusto. El papel especial de esta región de la corteza cerebral humana con relación al gusto umami, puede ser importante dado que es capaz de promover una amplificación no lineal de los estímulos del GMS e IMP ya combinados en los receptores del gusto, o puede ser que esta región de la corteza sea capaz de combinar la información a partir de vías umami parcialmente separadas para producir una amplia gama de respuestas a la combinación de GMS e IMP. Este último punto constituye un aspecto muy importante a ser estudiado en futuras investigaciones.

\section{UMAMI: UN DELLCIOSO SABOR FORMADO POR LA CONVERGENCIA DE LAS VÍAS DEL GUSTO Y DEL OLFATO EN EL CEREBRO HUMANO}

El glutamato no actúa sinérgicamente con otros gustos (dulce, salado, amargo y ácido) (Yamaguchi \& Kimizuka, 1979). Aún más, cuando el glutamato se presenta puro, como un estímulo al paladar, no tiene un gusto muy agradable (Beauchamp \& Pearson, 1991). La pregunta que surge es entonces ¿de qué forma el glutamato contribuye a la buena palatabilidad de los alimentos?. 
McCabe \& Rolls (2007) demostraron que cuando el glutamato es suministrado en combinación con un aroma (vegetal) armónico y sabroso, el sabor resultante puede ser mucho más agradable. Los autores, entonces, investigaron los mecanismos cerebrales subyacentes, teniendo en cuenta que el sabor se define como la combinación de gusto y aroma. Para que esta combinación (gusto y aroma) sea efectiva, las señales olfativas y gustativas deben ser transmitidas juntas. A partir de estudios en primates no humanos, se sabe que la corteza gustativa primaria en la ínsula anterior contiene neuronas que responden al gusto y textura de lo que ha sido colocado en la boca, pero que no responden al aroma (Verhagen et al., 2004). Tanto la corteza gustativa primaria como la corteza piriforme (olfatoria) se proyectan en dirección a la corteza orbitofrontal, y es allí donde se encuentran las neuronas bimodales gustativas y olfativas (Rolls \& Baylis, 1994). Estas redes de neuronas receptoras del sabor son desarrolladas a través del aprendizaje por asociación olfato-gusto (Critchley \& Rolls, 1996a; Rolls et al., 1996a). Se han identificado áreas olfativas en la corteza piriforme y en la corteza orbitofrontal (Zatorre et al., 1992; Rolls et al., 2003). Estudios acerca de la región donde la sensación gustativa y olfatoria se asocian en el cerebro humano (Small \& Prescott, 2005) han mostrado que, de hecho, existen áreas en la corteza orbitofrontal y en la ínsula anterior (agranular), que pueden ser activadas tanto por el gusto de la sacarosa como por el aroma de frutillas (fresas) (de Araujo et al., 2003b).

McCabe \& Rolls (2007) utilizaron un conjunto de estímulos diseñados para hacer posible que el gusto umami (producido por 0,1 M GMS y 0,005 M IMP) se probara solo o en combinación con un aroma vegetal sabroso de hortalizas. Esto permitió comparar los efectos de la combinación (GMSV en la Tabla 15.1) con los efectos de los componentes gustativos (GMS en la Tabla 15.1) u olfativos (t1V) suministrados separadamente. Para tener un estímulo de comparación, a fin de medir si los componentes gustativos y olfativos eran complementarios entre sí, el gusto umami fue presentado también en combinación con un aroma disonante, en este caso, una bebida alcohólica (ron). Los estímulos fueron suministrados oralmente en forma de solución insípida. Otra parte del diseño sirvió para tomar notas psicofísicas de los sujetos participantes en cada una de las pruebas a fin de realizar la clasificación, psicológicamente subjetiva, de los grados de placer, armonía y plenitud del sabor durante los experimentos de fMRI. De esta forma, fue posible correlacionar los efectos subjetivos de los estímulos, en cuanto al placer, con las señales BOLD, medidas en cada una de las pruebas. 
Tabla 15.1 - Estímulos y abreviaturas utilizados en la investigación de McCabe \& Rolls (2007) sobre el placer del sabor umami producido por la convergencia del gusto del glutamato monosódico y un aroma armónico.

\begin{tabular}{l|l}
\hline GMS & $0,1 \mathrm{M} \mathrm{GMS}+0,005 \mathrm{M}$ inosina-5'-monofosfato \\
GMSV & $0,1 \mathrm{M} \mathrm{GMS}+0,005 \mathrm{M}$ inosina-5'-monofosfato $+0,4 \%$ aroma vegetal \\
$\mathrm{NaCl}$ & $0,1 \mathrm{M} \mathrm{NaCl}$ \\
$\mathrm{NaClV}$ & $0,1 \mathrm{M} \mathrm{NaCl}+0,4 \%$ aroma vegetal \\
$\mathrm{GMSR}$ & $0,1 \mathrm{M} \mathrm{GMS}+0,005 \mathrm{M}$ inosina-5'- monofosfato $+2 \%$ aroma de ron \\
tl & $25 \mathrm{mM} \mathrm{KCl}+2,5 \mathrm{mM} \mathrm{NaHCO} 3$ (control insípido ) \\
tlV & $25 \mathrm{mM} \mathrm{KCl}+2,5 \mathrm{mM} \mathrm{NaHCO} 3+0,4 \%$ aroma vegetal \\
\hline
\end{tabular}

Fuente: McCabe \& Rolls, 2007.

Los niveles de placer, armonía y satisfacción del sabor se muestran en la Figura 15.3. La combinación de GMS y aroma vegetal fue clasificada como significativamente más agradable que el GMS puro $(\mathrm{p}<0,015)$. La combinación de GMS y aroma vegetal fue clasificada como más agradable que la combinación de $\mathrm{NaCl}$ y aroma vegetal (Figura 15.3). En un test de ANOVA de doble vía (F[1,11] $=22,05, \mathrm{p}<0,001)$, se demostró que el aumento en el grado de placer fue mayor cuando al aroma vegetal se le añadió GMS, que cuando se les añadió $\mathrm{NaCl}$. Este último, en verdad, produjo una disminución del placer (Figura 15.3). La adición de $\mathrm{NaCl}$ causó efectos de interacción similares a aquellos ocurridos al adicionar el aroma vegetal al GMS, en relación a la armonía $(F[1,11]=12,03 \mathrm{p}<0,005)$, y a la plenitud del sabor $(\mathrm{F}[1,11]=5,92, \mathrm{p}<0,03)$. 


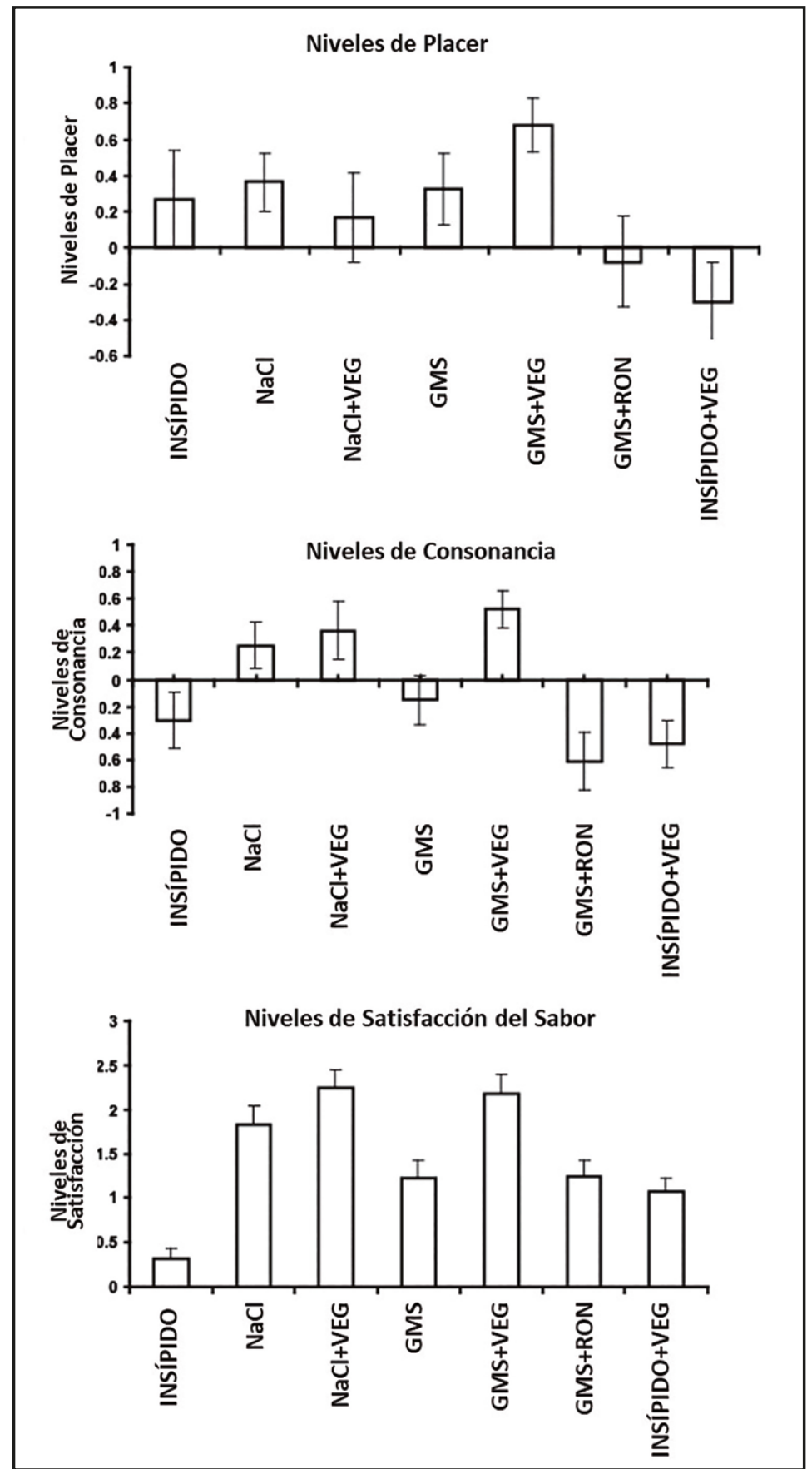

Figura 15.3 - Niveles de placer, armonía y plenitud de sabor (media $\pm \mathrm{DE})$ en la investigación sobre el placer del sabor umami, producido por la convergencia del gusto del GMS y un aroma armónico.

Fuente: McCabe \& Rolls, 2007. (umratings2.eps). 
Uno de los focos principales de las investigaciones de McCabe \& Rolls fue sobre la posibilidad de que la combinación del gusto de GMS con un aroma vegetal armónico, produjera activación selectiva de algunas regiones cerebrales. En este caso fueron utilizadas hortalizas como aroma vegetal armónico. Para probar esta hipótesis, se determinó por fMRI el contraste entre los efectos de la mezcla de GMS y verduras (GMSV), y la suma de las activaciones para GMS y verduras presentadas separadamente (Figura 15.4). Este contraste, entonces, es el de una adición supralineal, usada como un indicador de la interacción entre los componentes olfativos y gustativos, el cual revela un efecto altamente significativo en la corteza orbitofrontal medial centrado en ([-6 52 -14] Z=3,96), factor de corrección $\mathrm{p}=0,002$ ), que se extiende hasta la corteza cingulada progenual. Además, una parte del estriado ventral/tubérculo olfatorio, que recibe señales de la corteza orbitofrontal, mostró una activación supralineal significativa. Es de destacar que no hubo evidencia de esta supralinealidad en la ínsula gustativa, ni en la ínsula agranular. Los efectos supralineales fueron mucho menos (significativamente menos) evidentes para el cloruro de sodio y el sabor vegetal. Adicionalmente, la activación en estas regiones del cerebro fue correlacionada con el carácter agradable y satisfactorio del sabor, y con la armonía de los componentes del gusto y el olfato (McCabe \& Rolls, 2007).

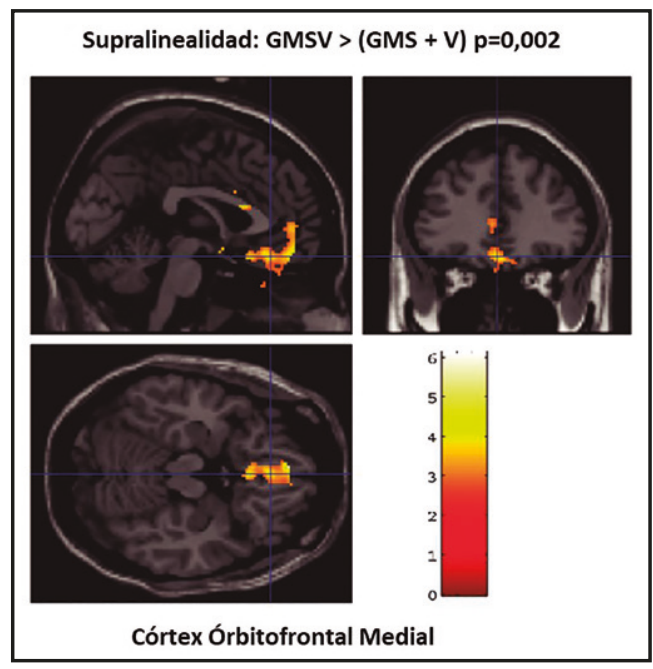

Figura 15.4 - Grado de adición entre GMS y un aroma vegetal armónico. Es presentado el contraste de la mezcla de GMS y aroma vegetal (GMSV), con la suma de las activaciones del GMS y el aroma vegetal presentados separadamente (GMS+V). Efecto altamente significativo en la corteza orbitofrontal centrado en [-6 52 -14] fc p $=0,002$, que con la activación se extendió a la corteza cingulada pregenual.

Fuente: McCabe \& Rolls, 2007. (SLMSGVofc2.eps). 
Se propuso entonces, que el glutamato actúa vía efectos no lineales en regiones multimodales de convergencia cortical entre gusto y aroma, cuando en combinación con un aroma armónico. De esta forma, se plantea la idea de que a nivel conceptual el umami puede ser pensado como un sabor rico y delicioso que se produce por una combinación del gusto del glutamato y un aroma agradable y armónico. El glutamato es, de este modo, un potenciador del sabor, debido a la forma como puede combinarse supralinealmente con aromas armónicos en áreas corticales, donde las vías gustativas y olfativas convergen mucho más allá de los receptores y donde el placer del sabor se representa.

\section{MODULACIÓN COGNITIVA DE RESPUESTAS EMOCIONALES AL SABOR Y GUSTO UMAMI}

Fue mencionado anteriormente que un factor importante en la palatabilidad del umami es la combinación del gusto del glutamato con un aroma complementario y armónico. Otro factor importante para hacer el umami agradable es el rótulo cognitivo o la descripción asociada, ya sea al gusto del glutamato o al sabor umami, tal como lo demostraron Grabenhorst et al. (2008a). El estímulo gustativo del GMS $(0,1 \mathrm{M})$ con IMP $(0,005 \mathrm{M})$ que produce el gusto umami, fue rotulado, a través de un estímulo visual, y calificado como "un gusto rico y delicioso" (GMS-"rico") o como "glutamato monosódico" considerado como básico (GMS-“básico”). De la misma forma, se rotuló el estímulo del sabor producido por la combinación del GMS con un aroma vegetal (GMSV-"rico"), en contraste con el "agua de hortalizas hervidas" (GMSV-"básico"). Al inicio del experimento, los sujetos de la investigación no fueron informados exactamente acerca de cuál estímulo gustativo o de sabor estaban colocando en la boca. La Figura 15.5 muestra que el GMS fue clasificado como significativamente más agradable cuando fue rotulado como "gusto rico y delicioso" que cuando fue rotulado como "glutamato monosódico" ( $\mathrm{p}=0,002)$. De manera similar, el GMSV fue considerado como significativamente más agradable cuando fue rotulado como "sabor rico y delicioso", que cuando se rotuló como "agua de hortalizas hervidas" ( $p=0,003)$. Adicionalmente, los rótulos no produjeron diferencias significativas en la clasificación de la intensidad de las mediciones del GMS. 


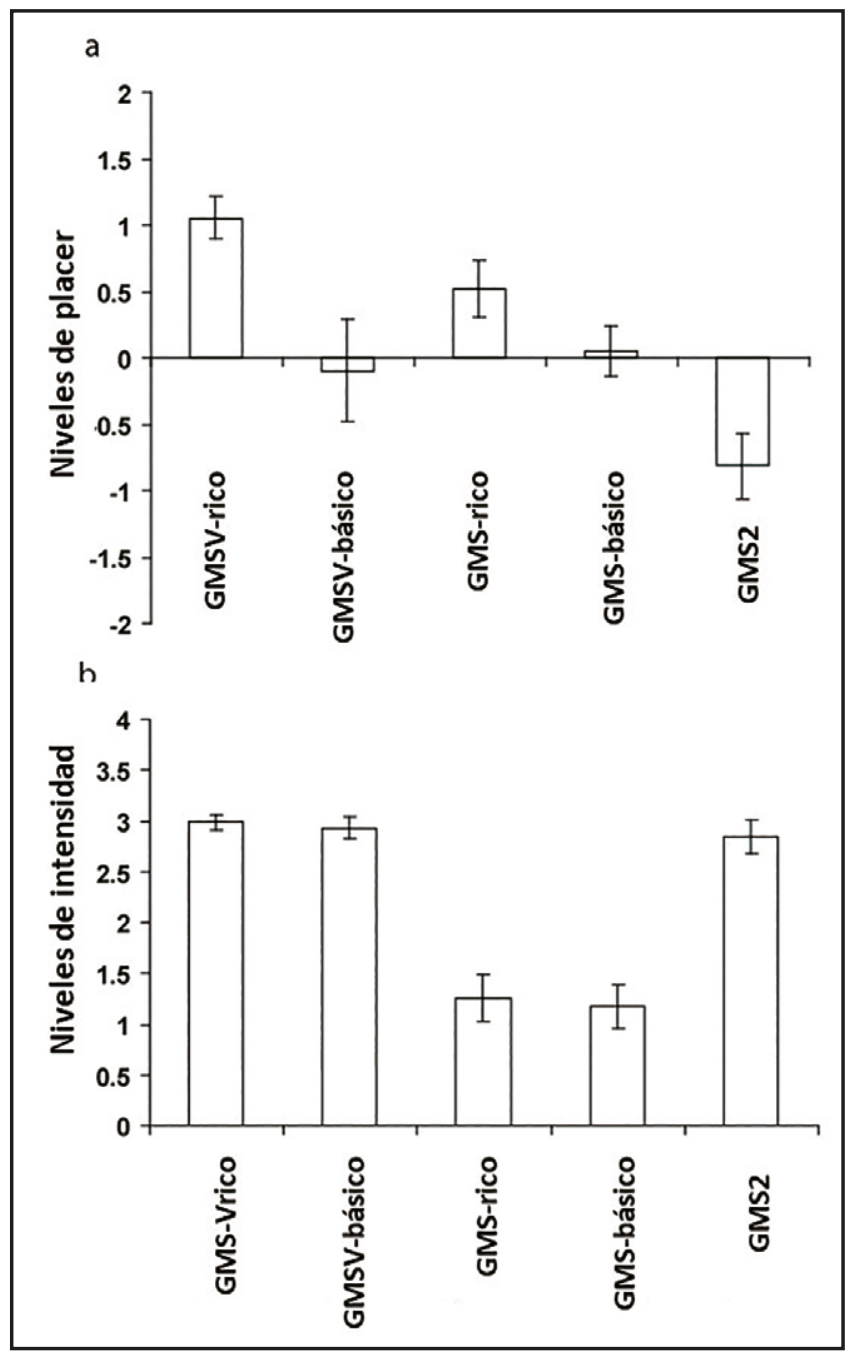

Figura 15.5 - Clasificación del placer (a) e intensidad (b) del gusto umami y del estímulo del sabor (media \pm DE). El estímulo gustativo del GMS fue clasificado como significativamente más agradable cuando se rotuló como "gusto rico y delicioso" que cuando se rotuló como "glutamato monosódico". De manera similar, el estímulo de sabor do GMSV se calificó como significativamente más agradable cuando se rotulaba como "sabor rico y delicioso" que cuando se rotulaba como "agua de hortalizas hervida". A diferencia de los efectos en los niveles de placer, los rótulos no produjeron diferencias significativas en los niveles de intensidad del gusto y el estímulo del sabor. Estos hallazgos proporcionan una clara evidencia de que los rótulos cognitivos modulaban la percepción del placer, pero no la intensidad del gusto y el sabor. El estímulo gustativo del GMS más concentrado (GMS2) se clasificó como significativamente menos agradable y más intenso que el estímulo gustativo rotulado idénticamente como GMS básico. En este caso, los rótulos eran idénticos ("glutamato monosódico"), y esto nos trae evidencia de que el gusto percibido también reflejaba las propiedades del estímulo (concentración baja versus alta).

Fuente: Grabenhorst et al., 2008a. (Ratingsreview.eps.). 
La comparación GMSV-"rico" vs GMSV-“básico" mostró efectos significativos en la corteza orbitofrontal medial. Esta región fue más fuertemente activada cuando el estímulo gustativo fue rotulado como "sabor rico y delicioso" que cuando fue rotulado como "agua de hortalizas hervidas" (en [-8 28 -20] Figura 15.6a, la señal BOLD, para las dos condiciones, se muestran en la Figura 15.6b, y los picos fueron significativamente diferentes como mostrado en la Figura 15.6c). Se observa, entonces, que las palabras en los rótulos influenciaron las activaciones producidas por el sabor umami en esta área. Aún más, las activaciones en la corteza orbitofrontal medial reflejaron el placer del estímulo, como mostrado por la correlación con los niveles subjetivos del placer causados por el estímulo del rotulado (GMSV-“rico", GMSV-“básico, GMS-“rico” y GMS-“básico) (La Figura 15.6d, muestra la señal BOLD en función de las clasificaciones). De este modo, en la corteza orbitofrontal, las activaciones generadas por los estímulos gustativos y de sabor fueron también moduladas por las palabras de los rótulos. Esta modulación cognitiva fue realizada a través de las activaciones relacionadas al placer generado por estos estímulos. Activaciones por el gusto del glutamato, promovidas e influencias por la terminología utilizada no rótulo, fueron moduladas en una región que recibe señales de la corteza orbitofrontal (la corteza cingulada pregenual), mientras que las activaciones por el gusto del glutamato como sabor umami fueron moduladas en otra región que recibe señales de la corteza orbitofrontal (el cuerpo estriado ventral). Una investigación relacionada demostró que los efectos de rótulos cognitivos top-down (de arriba para abajo) registrados solo en estas áreas eran relativamente débiles, y que los grandes efectos fueron registrados cuando había interacción entre los estímulos recibidos (de Araujo et al., 2005). Entonces, se puede entender que el mecanismo influye en la competencia sesgada de arriba hacia abajo, similar a la involucrada en la atención de arriba hacia abajo (Rolls \& Deco, 2002; Deco \& Rolls, 2003; Deco \& Rolls 2005; Rolls, 2008b). No se encontraron modulaciones cognitivas relacionadas con la emoción en la corteza gustativa insular (primaria), donde se representaba la intensidad, pero no el placer del gusto. Grabenhorst et al. (2008a) llegaron a la conclusión de que los efectos cognitivos en el nivel del lenguaje top-down alcanzan niveles muy bajos en las áreas corticales que representan el valor apetitivo del gusto y el sabor. Esta es una forma importante en la que la cognición influye en los mecanismos neuronales que controlan el placer del gusto del glutamato y del umami y, por lo tanto, el apetito. 
a
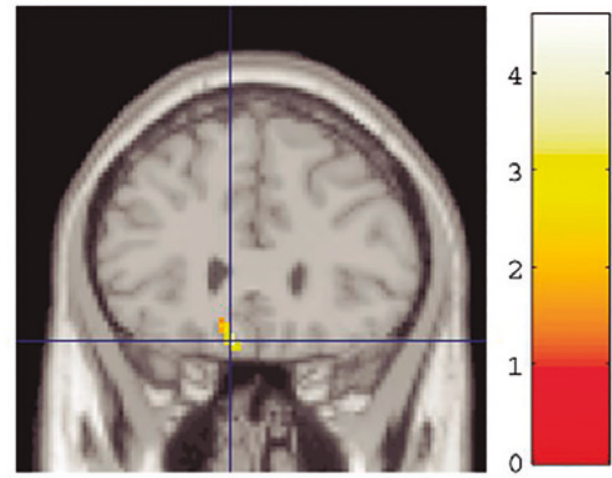

b

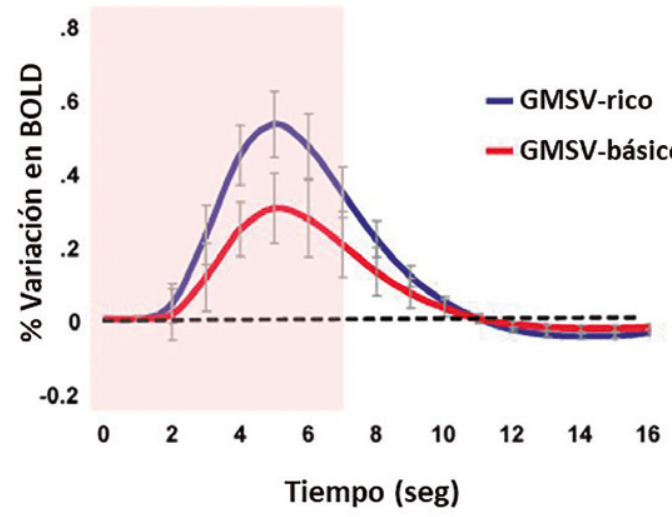

C

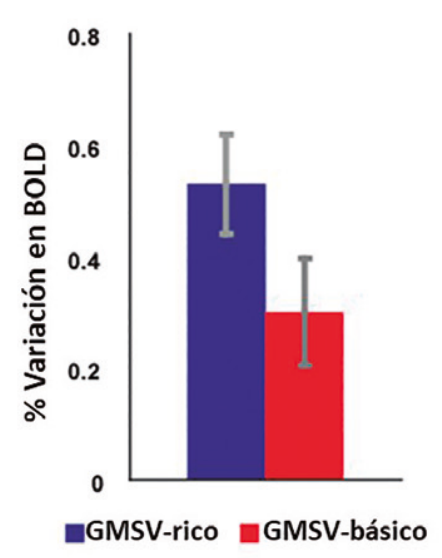

d

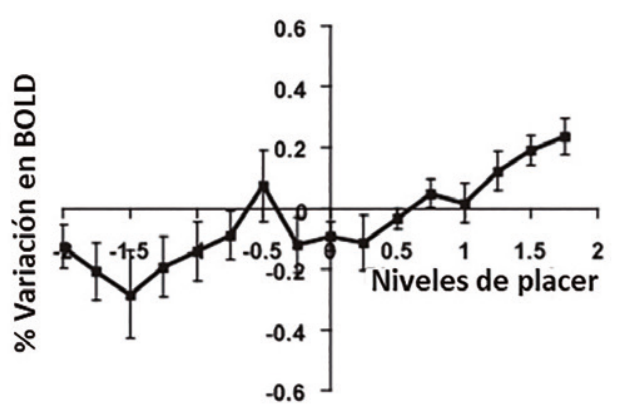

Figura 15.6 - (a) Fuerte activación de la corteza orbitofrontal medial cuando el estímulo gustativo fue rotulado como "sabor rico y delicioso" (GMSV-rico) en comparación cuando era rotulado como "agua de hortalizas hervida". (GMSV-básico) ([-8 28 -20]); (b). variación de las señales BOLD en función del tiempo para las dos condiciones estudiadas; (c). valores máximos de la señal BOLD (media entre individuos $\pm \mathrm{DP}$ ) fueron significativamente diferentes $(\mathrm{t}=3.06, \mathrm{df}=11$, $\mathrm{p}=0,01)$; (d). señal BOLD en la corteza orbitofrontal medial correlacionada con los niveles subjetivos del placer, del gusto y del sabor (media entre individuos $\pm \mathrm{DP}, \mathrm{r}=0,86, \mathrm{p}<0,001$ ).

Fuente: Grabenhorst et al., 2008a. (mOFC_Fig3review3.eps.).

\section{INFLUENCIA DE LAS PERCEPCIONES EN EL PROCESAMIENTO DEL}

\section{ESTÍMULO UMAMI}

¿Cómo las emociones influyen en el procesamiento del umami?. Grabenhorst \& Rolls (2008) demostraron que, cuando se presta atención al placer del 
gusto o sabor umami (en experimentos en que se le solicitó a cada participante, al inicio de la prueba, que clasificara el nivel de placer causado por los estímulos), el procesamiento en regiones del cerebro tales como la corteza orbitofrontal y corteza gustativa insular primaria se potencializa. Aún más, el placer subjetivo del gusto umami fue correlacionado con activaciones asociadas al gusto en la corteza orbitofrontal, mientras que la intensidad subjetiva del gusto umami fue correlacionada con activaciones en la corteza gustativa primaria. De este modo, dependiendo del contexto en el cual los gustos son presentados y de si el impacto emocional es relevante, el cerebro responde a un estímulo gustativo de forma diferente. Este sesgo diferencial de las regiones del cerebro involucradas en el procesamiento de un estímulo sensorial, dependiendo de cómo es la demanda cognitiva para eventos relacionados a las emociones versus el procesamiento de eventos relacionados a las sensaciones, puede ser un importante aspecto de cognición y atención. Esto tiene muchas implicaciones para la investigación y comprensión, tanto desde un enfoque psicológico como neurológico, de los efectos no solo del gusto, sino también de otros estímulos sensoriales, incluyendo el estímulo olfatorio (aromas) (Rolls et al., 2008). Adicionalmente, pone de manifiesto el valor de la atención selectiva ya que, cuando se evalúa el estímulo umami, puede ser muy importante el hecho de dirigir la atención hacia las propiedades físicas de los estímulos (como la intensidad), o al valor afectivo de los estímulos (como el placer). Aún más, los sistemas cerebrales participan de formas diferentes, en función de la dirección de estos dos tipos de atención.

\section{CONSIDERACIONES FINALES}

A nivel neuronal, existen representaciones del gusto del glutamato diferentes de las de otros estímulos gustativos, tanto en la corteza gustativa primaria como en la corteza orbitofrontal. También, algunas neuronas combinan el gusto de glutamato con estímulos olfatorios, visuales, y de textura y temperatura oral, y el valor de recompensa del gusto de glutamato es representado por neuronas en la corteza orbitofrontal. En humanos, el glutamato activa las cortezas gustativas primaria (insular), secundaria (orbitofrontal) y pregenual cingulada (terciaria), y la sensación de placer del umami es producida por la combinación del gusto del glutamato con un aroma armónico en áreas que van mucho más allá de los receptores del gusto umami, en la corteza orbitofrontal y en las cortezas cinguladas y pregenual. También, los efectos cognitivos alcanzan profundamente la corteza orbitofrontal, para influenciar el placer del sabor y del gusto umami. Si la atención se dirige a las propiedades físicas del umami o a su valor emocional, 
hay efectos moduladores top-down en diferentes sistemas de procesamiento cortical activados por el umami. De modo general, el procesamiento cortical para el sabor umami es importante, e a su vez permite entender cómo funciona el umami para promover un delicioso y rico sabor en los alimentos. Esto también tiene importantes implicaciones para entender el uso del sabor umami para promover una buena nutrición.

\section{REFERENCIAS BIBLIOGRÁFICAS}

BAYLIS, L. L. \& ROLLS, E. T. "Responses of neurons in the primate taste cortex to glutamate". Physiol Behav. 49(5): 973-979, 1991.

BAYLIS, L. L.; ROLLS, E. T. \& BAYLIS, G. C. “Afferent connections of the orbitofrontal cortex taste area of the primate". Neuroscience. 64(3): 801-812. 1995.

BEAUCHAMP, G. K. \& PEARSON, P. "Human development and umami taste". Physiol Behav. 49(5): 1009-1012, 1991.

CHAUDHARI, N.; LANDIN, A. M. \& ROPER, S. D. “A metabotropic glutamate receptor variant functions as a taste receptor". Nat Neurosci. 3(2): 113-119, 2000.

CRITCHLEY, H. D. \& ROLLS, E. T. "Olfactory neuronal responses in the primate orbitofrontal cortex: analysis in an olfactory discrimination task". $J$ Neurophysiol. 75(4):1659-1672, 1996a.

CRITCHLEY, H. D. \& ROLLS, E. T. "Hunger and satiety modify the responses of olfactory and visual neurons in the primate orbitofrontal cortex". J Neurophysiol. 75(4): 1673-1686, 1996b.

DE ARAUJO, I. E. T. et al. "Representation of umami taste in the human brain". J Neurophysiol. 90(1):313-319, 2003 a.

DE ARAUJO, I. E. T. et al. "Taste-olfactory convergence, and the representation of the pleasantness of flavour, in the human brain". Eur J Neurosci. 18(7): 2059-2068, 2003b.

DE ARAUJO, I. E. T. et al. "Cognitive modulation of olfactory processing". Neuron. 46(4): 671-679, 2005.

DECO, G. \& ROLLS, E. T. “Attention and working memory: a dynamical model of neuronal activity in the prefrontal cortex". Eur J Neurosci. 18(8): 2374-2390, 2003. 
DECO, G. \& ROLLS, E. T. "Neurodynamics of biased competition and co-operation for attention: a model with spiking neurons". J Neurophysiol. 94(1): 295313, 2005.

GRABENHORST, F. \& ROLLS, E. T. "Selective attention to affective value alters how the brain processes taste stimuli”. Eur J Neurosci. 27(3): 723-729, 2008.

GRABENHORST, F.; ROLLS, E. T. \& BILDERBECK, A. "How cognition modulates affective responses to taste and flavor: top-down influences on the orbitofrontal and pregenual cingulate cortices". Cereb Cortex. 18(7): 1549-1559, $2008 \mathrm{a}$.

GRABENHORST, F.; ROLLS, E. T. \& PARRIS, B. A. "From affective value to decision-making in the prefrontal cortex". Eur J Neurosci. 28: 1930-1939, 2008b.

KADOHISA, M.; ROLLS, E. T. \& VERHAGEN, J. V. "Neuronal representations of stimuli in the mouth: the primate insular taste cortex, orbitofrontal cortex, and amygdale”. Chem Senses. 30(5): 401-419, 2005.

KAWAMURA, Y. \& KARE, M. R. Umami: a basic taste. New York, Dekker, 1987.

MARUYAMA, Y. et al. "Umami responses in mouse taste cells indicates more than one receptor". J Neurosci. 26(8): 2227-2234, 2006.

MCCABE, C. \& ROLLS, E. T. "Umami: a delicious flavor formed by convergence of taste and olfactory pathways in the human brain". Eur J Neurosci. 25(6):1855-1864, 2007.

NORGREN, R. "Central neural mechanisms of taste". In: DARIEN-SMITH, I. Handbook of physiology - The nervous system III. Sensory processes 1. Washington, American Physiological Society, 1984, pp. 1087-1128.

O'DOHERTY, J. et al. "The representation of pleasant and aversive taste in the human brain”. J Neurophysiol. 85(3): 1315-1321, 2001.

RIFKIN, B. \& BARTOSHUK, L. M. "Taste synergism between monosodium glutamate and disodium 5'-guanylate”. Physiol Behav. 24(6): 1169-1172, 1980.

ROLLS, E. T. "The representation of umami taste in the taste cortex". J Nutrit. 130(4S Supp1): S960-S965, 2000.

ROLLS, E. T. "The representation of umami taste in the human and macaque cortex”. Sensory Neuron. 3(3): 227-242, 2001. 
ROLLS, E. T. "Brain mechanisms that analyse umami taste and their relation to the control of feeding". In: ELMADFA, I.; ANKLAM, E. \& KONIG, J. S. Forums in nutrition 56: Modern aspects of nutrition - Present knowledge and future perspectives. Basel, Karger, 2003, pp. 84-87.

ROLLS, E. T. Emotion explained. Oxford, Oxford University Press, 2005.

ROLLS, E. T. "Understanding the mechanisms of food intake and obesity". Obes Rev. 8(Suppl 1): 67-72, 2007a.

ROLLS, E. T. "Sensory processing in the brain related to the control of food intake". Proc Nutr Soc. 66(1): 96-112, 2007b.

ROLLS, E. T. "Functions of the orbitofrontal and pregenual cingulate cortex in taste, olfaction, appetite and emotion". Acta Physiol Hung. 95(2): 131-164, $2008 \mathrm{a}$.

ROLLS, E. T. Memory, attention, and decision-making: a unifying computational neuroscience approach. Oxford, Oxford University Press, 2008b.

ROLLS, E. T. "From reward value to decision-making: neuronal and computational principles". In: DREHER, J. C. \& TREMBLAY, L. (ed.). Reward and decision-making. Amsterdam, Elsevier, 2009.

ROLLS, E. T. \& BAYLIS, L. L. "Gustatory, olfactory, and visual convergence within the primate orbitofrontal cortex". J Neurosci. 14(9): 5437-5452, 1994.

ROLLS, E. T. \& DECO, G. Computational neuroscience of vision. Oxford, Oxford University Press, 2002.

ROLLS, E. T. \& SCOTT, T. R. "Central taste anatomy and neurophysiology". In: DOTY, R. L. (ed.). Handbook of olfaction and gustation. 2. ed. New York, Dekker, 2003, pp. 679-705.

ROLLS, E. T. \& GRABENHORST, F. "The orbitofrontal cortex and beyond: from affect to decision-making". Progress in Neurobiology. 86(3): 216-244, 2008.

ROLLS, E. T.; SIENKIEWICZ, Z. J. \& YAXLEY, S. "Hunger modulates the responses to gustatory stimuli of single neurons in the caudolateral orbitofrontal cortex of the macaque monkey". Eur J Neurosci. 1(1): 53-60, 1989.

ROLLS, E. T.; YAXLEY S. \& SIENKIEWICZ, Z. J. "Gustatory responses of single neurons in the caudolateral orbitofrontal cortex of the macaque monkey". J Neurophysiol. 64: 1055-1066, 1990. 
ROLLS, E. T.; KRINGELBACH, M. L. \& DE ARAUJO, I. E. T. "Different representations of pleasant and unpleasant odors in the human brain". Eur $J$ Neurosci. 18(3): 695-703, 2003.

ROLLS, E. T. et al. "The responsiveness of neurones in the frontal opercular gustatory cortex of the macaque monkey is independent of hunger". $J$ Physiol. 397: 1-12, 1988.

ROLLS, E. T. et al. "Orbitofrontal cortex neurons: role in olfactory and visual association learning”. J Neurophysiol. 75(5): 1970-1981, 1996 a.

ROLLS, E. T. et al. "Responses of neurons in the primate taste cortex to the glutamate ion and to inosine 5 '-monophosphate". Physiol Behav. 59(4-5): 9911000, $1996 b$.

ROLLS, E. T. et al. "Selective attention to affective value alters how the brain processes olfactory stimuli”. J Cogn Neurosci. 20(10): 1815-1826, 2008.

SCOTT, T. R. \& PLATA-SALAMAN, C. R. "Taste in the monkey cortex". Physiol Behav. 67(4): 489-511, 1999.

SCOTT, T. R. et al. "Gustatory responses in the frontal opercular cortex of the alert cynomolgus monkey”. J Neurophysiol. 56(3): 876-890, 1986.

SMALL, D. M. \& PRESCOTT, J. "Odor/taste integration and the perception of flavor”. Exp Brain Res. 166(3-4): 345-357, 2005.

SMALL, D. M. et al. "Human cortical gustatory areas: a review of functional neuroimaging data". Neuro Report. 10(1): 7-14, 1999.

VERHAGEN, J. V.; KADOHISA, M. \& ROLLS, E. T. “The primate insular/ opercular taste cortex: neuronal representations of the viscosity, fat texture, grittiness, temperature and taste of foods". J Neurophysiol. 92(3): 1685-1699, 2004.

WILSON, J. L. et al. "Fast, fully automated global and local magnetic field optimisation for fMRI of the human brain". Neuroimage. 17(2): 967-976, 2002.

YAMAGUCHI, S. "The synergistic taste effect of monosodium glutamate and disodium 5'-inosinate". J Food Sci. 32(4): 473-478, 1967.

YAMAGUCHI, S. \& KIMIZUKA, A. "Psychometric studies on the taste of monosodium glutamate". In: FILER, L. J. et al. Glutamic Acid: Advances in Biochemistry and Physiology. New York, Raven Press, 1979, pp. 35-54. 
YAMAGUCHI, S. \& NINOMIYA, K. "Umami and food palatability". J Nutrit. 130(4S Suppl): 921S-926S, 2000.

YAXLEY, S.; ROLLS, E. T. \& SIENKIEWICZ, Z. J. “The responsiveness of neurons in the insular gustatory cortex of the macaque monkey is independent of hunger". Physiol Behav. 42(3): 223-229, 1988.

YAXLEY, S.; ROLLS, E. T. \& SIENKIEWICZ, Z. J. “Gustatory responses of single neurons in the insula of the macaque monkey". $J$ Neurophysiol. 63(4):689-700, 1990.

ZATORRE, R. J. et al. "Functional localization of human olfactory cortex". Nature. 360(6402): 339-340, 1992.

ZHAO, G. Q. et al. "The receptors for mammalian sweet and umami taste”. Cell. 115(3): 255-266, 2003. 